\title{
Some New Properties of the Triangle.
}

By J. S. Mackay, M.A., I.L.D.

[The substance of this communication will be included in $\mathrm{Dr}$

Mackay's paper on The Triangle in the first volume of the Proceedings, which is about to be printed.]

\section{Proofs of some optical theorems. \\ By William Peddie, D.Sc.}

[The results of this paper will be contained in Dr Peddie's book on Physics, which will appear in a short time.]

Second Meeting, December 12th, 1890.

R. E. Allardice, Esq., President, in the Chair.

On the condition that the straight line

$$
l x+m y+n z=0
$$

should be a normal to the conic

$$
(a, b, c, f, g, h)(x, y, z)^{2}=0
$$

the co-ordinates being trilinear.

\section{By R. H. Pinkerton, M.A.}

1. The condition in question may be found by using the following theorem :-

If the equation in trilinear co-ordinates

$$
\mathrm{F}(x, y, z) \equiv\left(u, v, w, u^{\prime}, v^{\prime}, w^{\prime}\right)(x, y, z)^{2}=0 \quad . .
$$

represents a pair of straight lines, then the line whose equation is

$$
l x+m y+n z=0 \quad \ldots \quad \ldots \quad \ldots
$$

will be perpendicular to one of those lines if $\mathrm{F}(l-m \cos \mathrm{C}-n \cos \mathrm{B}, m-n \cos \mathrm{A}-l \cos \mathrm{C}, n-l \cos \mathrm{B}-m \cos \mathrm{C})=0$ where $\mathrm{A}, \mathrm{B}, \mathrm{C}$ are the angles of the fundamental triangle. 
To prove this, transform the equations (A) and (B) to Cartesian co-ordinates by writing, as usual, $x, y, \quad z=x \cos \alpha+y \sin \alpha-p_{1}, \quad x \cos \beta+y \sin \beta-p_{2}, \quad x \cos \gamma+y \sin \gamma-p_{3}$, where $\beta-\gamma=180^{\circ}-\mathrm{A}$, etc.

The equation (A) thus becomes in Cartesian co-ordinates

$F\left(x \cos \alpha+y \sin \alpha-p_{1}, x \cos \beta+y \sin \beta-p_{2}, x \cos \gamma+y \sin \gamma-p_{3}\right)=0$, and the equation to the pair of straight lines through the origin of co-ordinates parallel to the lines $(\mathrm{A})$, is

$\mathrm{F}(x \cos \alpha+y \sin \alpha, x \cos \beta+y \sin \beta, x \cos \gamma+y \sin \gamma)=0 \quad \ldots \quad\left(\mathrm{A}^{\prime}\right)$.

The equation in Cartesian co-ordinates to the straight line through the origin parallel to the $(B)$ is similarly

$$
\lambda x+\mu y=0 \quad \ldots \quad \ldots \quad \ldots \quad \ldots
$$

where $\lambda, \mu=l \cos \alpha+m \cos \beta+n \cos \gamma, l \sin \alpha+m \sin \beta+n \sin \gamma$.

Now the line (B) will be perpendicular to one of the lines $(\dot{A})$ if the line $\left(B^{\prime}\right)$ is perpendicular to one of the lines $\left(A^{\prime}\right)$. The condition that $\left(B^{\prime}\right)$ should be perpendicular to one of the lines $\left(A^{\prime}\right)$ is found by substituting in the equation $\left(A^{\prime}\right) \lambda, \mu$ for $x, y$. The line (B) will therefore be perpendicular to one of the lines (A) if

$\mathrm{F}(\lambda \cos \alpha+\mu \sin \alpha, \lambda \cos \beta+\mu \sin \beta, \lambda \cos \gamma+\mu \cos \gamma)=0$.

Replacing $\lambda, \mu$ by their values in terms of $l, m, n$, we get

$$
\begin{aligned}
\lambda \cos \alpha+\mu \cos \beta & =\cos \alpha(l \cos \alpha+m \cos \beta+n \cos \gamma) \\
& +\sin \alpha(l \sin \alpha+m \sin \beta+n \sin \gamma) \\
& =l+m \cos (\alpha \sim \beta)+n \cos (\gamma \sim \alpha) \\
& =l-m \cos \mathrm{C}-n \cos \mathrm{B},
\end{aligned}
$$

with similar values $\lambda \cos \beta+\mu \sin \beta$ and $\lambda \cos \gamma+\mu \sin \gamma$. Hence the theorem follows.

2. Taking now the conic

$$
\mathrm{S} \equiv(a, b, c, f, g, h)(x, y, z)^{2}=0
$$

and the straight line

$$
l x+m y+n z=0 \quad \ldots \quad \ldots \quad \ldots \quad(\mathrm{P}),
$$

we write down the equation to the pair of tangents to the conic at the points where the straight line cuts the conic. This equation is

$$
\mathrm{S} \Sigma=\Delta(l x+m y+n z)^{2} \quad \ldots \quad \ldots \quad(\mathrm{T}),
$$

where $\mathrm{Z}$ is written for $(\mathrm{A}, \mathrm{B}, \mathrm{C}, \mathrm{F}, \mathrm{G}, \mathrm{H})(l, m, n)^{2}$, and $\Delta, \mathrm{A}, \mathrm{B}, \mathrm{C}$, $\mathrm{F}, \mathrm{G}, \mathbf{H}$ have their usual meanings.

The line $(\mathrm{P})$ will be a normal to the conic $\mathrm{S}$ if it is perpendicular to one of the lines $(\mathrm{T})$. The condition for this is, by (C), found by 
substituting $l-m \cos \mathrm{C}-n \cos \mathrm{B}$, etc., for $x, y, z$ in $(\mathrm{T})$. The result is $(a, b, c, f, g, h)(l-m \cos \mathrm{C}-n \cos \mathrm{B}, m-n \cos \mathrm{A}-l \cos \mathrm{C}, n-l \cos \mathrm{B}-m$ $\cos \mathrm{C})^{2} \times \Sigma=\Delta\left(l^{2}+m^{2}+n^{2}-2 m n \cos \mathrm{A}-2 n l \cos \mathrm{B}-2 l m \cos \mathrm{C}\right)^{2}$, the condition sought for.

The triangle and its escribed parabolas.

By A. J. Pressiand, M.A.

$\S 1$. The problem "to inflect a straight line between two sides of a triangle so that the intercepted portion is equal to the segments cut off" has been discussed in the third volume of the Proceedings.

If we discuss the same analytically; taking $\mathrm{CB}$ and $\mathrm{CA}$ as axes of $x$ and $y$ (Fig. 1) and calling each segment $k$, the equation of the line considered is

$$
x /(a-k)+y /(b-k)=1, \quad \ldots \quad \ldots \quad(a)
$$

where

The envelope of $(\alpha)$ considering $k$ unrestricted by $(\beta)$ is

$$
(x+y)^{2}-2(a-b)(x-y)+(a-b)^{2}=0 \quad \ldots
$$

a parabola touching the axis of $x$ at $(a-b, 0)$

$$
\text { and the axis of } y \text { at }(0, b-a)
$$

and which can be shown to touch $A B$

at the point $\left(\frac{a^{2}}{a-b},-\frac{b^{2}}{a-b}\right)$.

Its axis is

$$
x+y=0
$$

and tangent at vertex $x-y=\frac{a-b}{2}$.

§2. If we consider $x /(a-k)+y /(b+k)=1$

which cuts off equal portions from $\mathrm{BC}$ and $\mathrm{CA}$ produced, the envelope is

$$
(x-y)^{2}-2(a+b)(x+y)-(a+b)^{2}=0 .
$$

which touches $\mathbf{C B}$ at $(a+b, 0) \quad$ the point $l$,

$$
\begin{aligned}
& \mathrm{CA} \text { at }(0, a+b) \quad \text { the point } k, \\
& \mathrm{AB} \text { at }\left(\frac{a^{2}}{a+b}, \frac{b^{2}}{a+b}\right) \text { the point } t,
\end{aligned}
$$

the axis being

$$
\begin{aligned}
& x-y=0 \\
& x+y=\frac{a+b}{2} .
\end{aligned}
$$

and tangent at vertex 